\title{
Classical conditioning of attitudes as a function of number of persuasion trials and argument (UCS) strength'
}

ROBERT FRANK WEISS, LEO M. CHALUPA, BERNARD S. GORMAN, AND NEIL H. GOODMAN, DEPARTMENT OF PSYCHOLOGY, QUEENS COLLEGE OF THE CITY UNIVERSITY OF NEW YORK, Flushing, N. Y. 11367

Conditioned attitude strength was an increasing function of number of persuasion trials $(1$ vs 3 exposures to communication $)(p<.02)$ and the strength of the opinion-eliciting argument $(p<.001)$ with these two variables interacting multiplicatively $(p<.05)$ to determine attitude strength $(N=200)$. Results were predicted from an extension of Hull-Miller-Spence learning theory.

Recent studies (e.g., Weiss, 1967; Weiss et al, 1963) have tended to support the application of instrumental conditioning and selective learning models to attitude formation (Weiss, 1962). The present paper explores the possibilities of a classical conditioning model (Weiss, 1962) in which the opinion to be learned is the conclusion of a syllogism. The Ss are exposed to a persuasive communication which includes the premises of the syllogism, but not the conclusion, and $S$ is left to draw the implied conclusion for himself. Figure 1 shows corresponding paradigms for a classical conditioning trial and a persuasion trial.

Research based on this classical conditioning model requires communications which incorporate two elements: (1) the Opinion-Eliciting Argument (syllogism premises); and (2) the Cue Statement, two (neutral) words which immediately precede the opinion-eliciting argument and will later constitute part of the test used to measure attitude acquisition. The cue statement precedes the opinion-eliciting argument, so that an S listening to (or reading) the communication will first hear the cue statement, followed by the opinion-eliciting argument (syllogism premises), and then draw the conclusion implied by the argument. This sequence of events may be regarded as analogous to the sequence: CS, UCS, UR. The cue statement is the CS, and the opinioneliciting argument is the UCS which elicits the implied opinionthe UR. Through repetition of the sequence the implied opinion (UR) becomes conditioned to the cue statement (CS) and thus becomes a conditioned opinion (CR) as shown in Fig. 1 .

Following the logic of this paradigm further, the number of repetitions of this sequence is analogous to the number of conditioning trials, and the power of the opinion-eliciting argument to convincingly imply the opinion is analogous to the strength of the UCS. Research and theory in classical conditioning (e.g., Spence, 1956; Spence \& Platt, 1966) indicate that CR strength is an increasing function of trials and UCS-strength, and that these two variables combine multiplicatively (D $\times H$ ) to determine CR strength. Theoretically, then, attitude strength should be an increasing function of persuasion trials and argument strength, and these two variables should combine multiplicatively to determine conditioned attitude strength.

Method and Procedure. The Ss were 200 undergraduates. The design was a 2 by 2 factorial with $40 \mathrm{Ss}$ in each of the four cells. There were two levels of number of persuasion trials (one vs three readings of the persuasive communications) and two levels of argument strength. There was also a no-persuasion control group $(N=40)$ which was exposed to the same buffer communications as the experimental groups, but not to the persuasive communications.
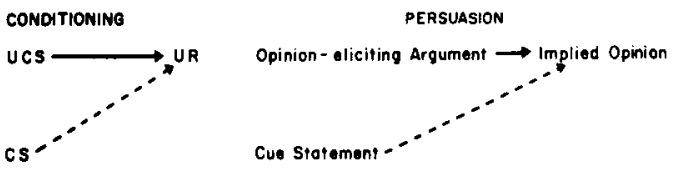

Fig. 1. Corresponding paradigms for a classical conditioning trial and a persuasion trial.
Under the impression that he was participating in a study of "speech patterns and decision-making" each experimental $S$ read three persuasive communications and three buffers into a tape recorder. One-trial Ss read each communication once and 3-trial Ss read each communication three times. There were two reading sessions within the $30-50 \mathrm{~min}$ experimental period, each followed immediately by attitude measurement. The first reading session covered two persuasive communications and two buffers, and the second, one persuasive and one buffer.

In order to study "conditioning" rather than "habit reversal" the persuasive communications were directed at fictitious opinion topics, on which $96-98 \%$ of a sample of 100 similar undergraduates had no initial opinion (e.g., Bayzin was considerate of his troops). The Bayzin communication concerned a (fictitious) 13 th century Turkish general, and the premises (argued with different strength for the two argument conditions) were: Great generals are considerate of their troops, and Bayzin was a great general. The cue statement was the two words immediately preceding the argument (e.g., Asia Minor).

The attitude measuring apparatus assessed each S's probability and latency of agreement with the opinion after $S$ had been exposed to persuasive communication. The cue statement and opinion were projected on a screen (preceeded by 11-13 buffers), and S signified his agreement (if he agreed) by moving a lever toward the opinion. When an opinion was projected on the screen, an electric timer automatically began to measure latency of agreement (to $1 / 100 \mathrm{sec}$ ). When the lever was moved $1 / 4 \mathrm{in}$., a photobeam silently stopped the timer (speed $=1 /$ latency). The timing equipment was not visible to the Ss, who did not know they were being timed. If an $\mathbf{S}$ did not respond within $40 \mathrm{sec}$, his speed of agreement with that opinion was considered to be zero. The Ss who did not agree did not move the lever. The mean of the three speed scores was computed for each S. Probability of agreement was computed for each $S$ as agreements/3, and then transformed into excitatory potential (E) in sigma-units (Hull, 1943).

Results. The relationship between speed and $\mathrm{E}$ was analyzed by orthogonal polynomials. Speed was a linear function of $E$. The linear trend was highly significant $(F=394.26, p<.001)$ and accounted for $99.75 \%$ of the between variance, leaving a negligible

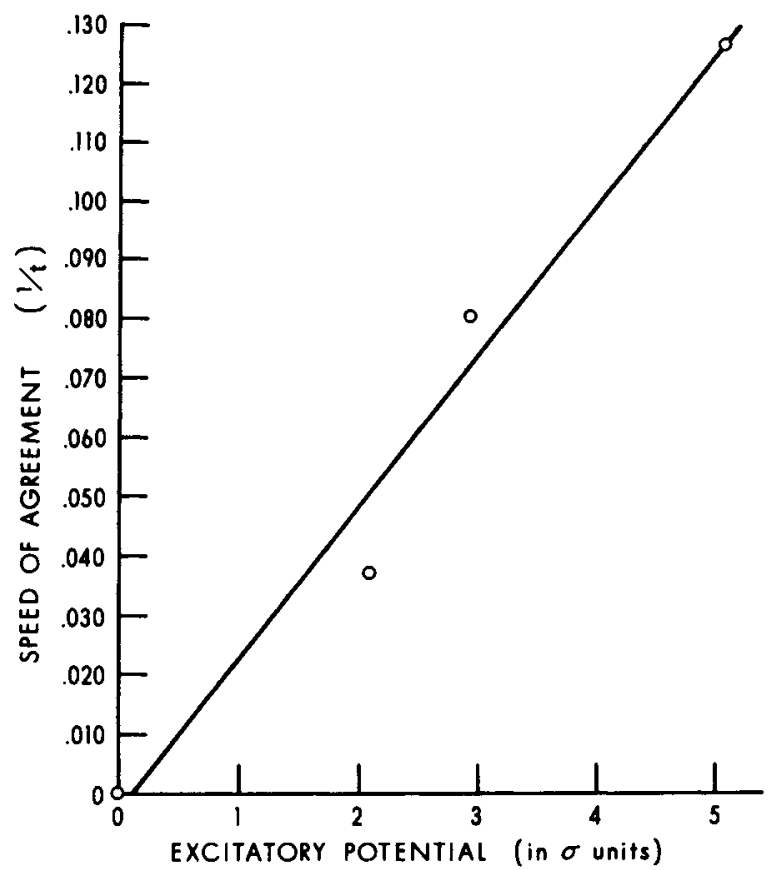

Fig. 2. Speed (1/latency) of agreement as a function of excitatory potential (E) 


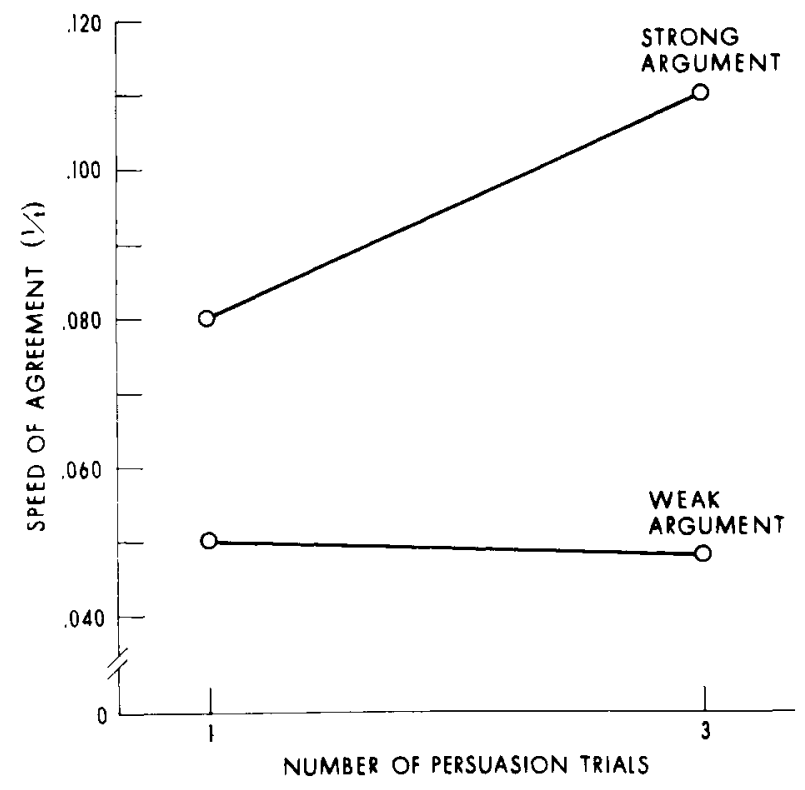

Fig. 3. Speed (1/latency) of agreement as a function of number of persuasion trials at two levels of argument (UCS) strength.

nonlinear residual $(F<1)$. A least-squares fit is shown in Fig. 2. This linearity permits the theoretical $\mathrm{D} \times \mathrm{H}=\mathrm{E}$ relationship to appear undistorted in the speed results.

The diverging curves in Fig. 3 show how Argument strength and Trials combine multiplicatively to determine mean speed (Interaction $F=4.40$, $\mathrm{df}=1 / 156, \mathrm{p}<.05)$. Speed was an increasing function of argument strength $(F=38.10, \mathrm{df}=1 / 156, \mathrm{p}<.001)$. Speed increased from one to three trials only for the strong argument groups (Mann-Whitney $z=2.10, p<.02$ ), although speed did increase from 0-trials (control) to 1 trial of weak argument $(\mathrm{z}=6.06, \mathrm{p}<.0001)$ indicating that the weak argument communication was effective. Speed and $E$ measures yielded similar results with speed being more sensitive. The weak-argument groups decreased, nonsignificantly, in speed from one to three trials but increased, nonsignificantly, in E.

Discussion. The research reported here derives from a theory of attitude learning (Weiss, 1962) which is based on systematic analogies with learning research and Hull-Miller-Spence learning theory. The theory was formulated in sufficient detail to permit derivations which are not always intuitively obvious, such as interaction effects and curve shapes. Thus the theory predicted not only the main effects of the trials and argument strength variables, but also the multiplicative interaction between them. The results of another study also support the theory: as in classical conditioning, probability was a negatively accelerated increasing function of another UCS-strength variable, source consensus (Weiss, et al, 1967). Results supporting the theory have also been found in "instrumental attitude learning," such as persuasion analogs of the delay of reinforcement gradient (Weiss, 1967) and the additive drive-reward interaction (Weiss, et al, 1963).

\section{REFERENCES}

HULL, C. L. Principles of behavior. New York: Appleton-Century, 1943.

SPENCE, K. W. Behavior theory and conditioning. New Haven: Yale Univ. Press, 1956.

SPENCE, K. W., \& PLATT, J. R. UCS intensity and performance in eyelid conditioning. Psychol. Bull., 1966, 65, 1-10.

WEISS, R. F. Persuasion and the acquisition of attitudes: models from conditioning and selective learning. Psychol. Rep., 1962, 11, 709-732.

WEISS, R. F., A delay of argument gradient in the instrumental conditioning of attitudes. Psychon. Sci., 1967, 8, 475-458.

WEISS, R. F., RAWSON, H. E., \& PASAMANICK, B. Argument strength, delay of argument, and anxiety in the "conditioning" and "selective learning" of attitudes. J. abnorm. soc. Psychol., 1963, 67, 157-165.

WEISS, R. F., WEISS, J. J., \& CHALUPA, L. M. Classical conditioning of attitudes as a function of source consensus. Psychon. Sci., 1967, 9, $465-466$.

\section{NOTE}

1. Research supported by NIMH Grant MH-12402. 